\title{
Passive UHF RFID Yarn For Temperature Sensing Applications
}

\author{
Sofia Benouakta \\ Univ Lyon, Université Claude Bernard \\ Lyon 1, INSA Lyon, Ecole Centrale de \\ Lyon, CNRS, Ampère,UMR5505, 69622 \\ Villeurbanne, France \\ sofia.benouakta@univ-lyon1.fr
}

\author{
Florin Hutu \\ Université de Lyon \\ INSA Lyon, Inria, CITI \\ Villeurbanne, France \\ florin-doru.hutu@insa-lyon.fr
}

\author{
Yvan Duroc \\ Univ Lyon, Université Claude Bernard \\ Lyon 1, INSA Lyon, Ecole Centrale de \\ Lyon, CNRS, Ampère,UMR5505, 69622 \\ Villeurbanne, France \\ yvan.@univ-lyon1.fr
}

\begin{abstract}
This paper presents an Ultra High Frequency (UHF) Radio Frequency IDentification (RFID) temperature sensor integrated into a textile yarn. The tag system consists of a thermistor of which the resistance is sensitive to temperature variation. The theoretical working principle is detailed and validated using electromagnetic simulation. The UHF RFID temperature sensor is fabricated using the E-Thread ${ }^{\circledR}$ technology. Experimental results demonstrate the potential of the sensor for detecting a temperature variation comprised between $25^{\circ} \mathrm{C}$ and $70^{\circ} \mathrm{C}$ through a variation of the reading distance between 4.5 meters to 6.2 meters.
\end{abstract}

Keywords-RFID sensor, textile yarn, E-Thread ${ }^{\circledR}$, RFID tag

\section{INTRODUCTION}

The Radio Frequency Identification (RFID) technology became ubiquitous in our everyday life [1]. RFID is used in a wide range of applications such as logistics, access control and ID cards. Passive RFID systems consist on a communication between two elements: a reader and a passive tag by using the backscattering technique [2].

More recently, passive RFID tags operating in the UHF RFID band have gained interest in the wearable concept. For example, the association of an RFID tag to an accessory or a clothing allows its identification from a distance that can reach several meters. However, identification is not the only capability of an RFID tag and several research works have demonstrated their potential of being employed as sensors [3]. Several examples could be found in the literature where the RFID tags are made by using different techniques: for instance, a temperature sensor tag fabricated using 3D printing technique [4]; a humidity sensor tag fabricated using the inkjet printing technique and Kapton as a sensitive material [5]; a strain sensor tag fabricated using adhesive conductive textile in order to associate the antenna to a textile substrate [6]. In these studies, it is worth to note that the tag and the object are considered as two individual elements.

The concept presented in [7] consists of an RFID tag integrated into a textile yarn using the new assembling technology, i. e. E-Thread $\AA$ [8]. The RFID yarn allows every object integrating it to be identified. Inspired from this design, [9] emphasized the potential of the RFID yarn for sensing applications. In this paper, we propose the design of a passive RFID yarn including the temperature sensing functionality. The proposed structure of the sensor tag uses a thermistor as sensitive element and exploits the impedance mismatching between the antenna and the chip to translate the temperature variation.
Section II details the configuration and the theoretical concept of the designed temperature sensor-tag yarn. Section III presents the design parameters and the main simulated characteristics. Section IV shows the experimental tests in order to validate the proposed concept. Finally, section V draws the conclusion and perspectives.

\section{Principle Of The TEMPERATURe SENSOR-TAG YARN}

\section{A. Design and Configuration}

The proposed sensor tag (Fig. 1) relies on the E-Thread $\AA$ UHF RFID yarn structure presented in [7]. The tag is composed of a radiating element having the length $L_{1}$ associated to an RFID chip [10]. The RFID chip is connected to a stub of length $L_{2}$. Both the radiating element and the stub are forming a transmission line where the conductors are of radius $a$ and $a$ ' respectively. The conductors are separated by a distance $s$ (center to center). The stub is loaded by a thermistor that has as an electrical equivalent model a variable resistance $R_{t h}$. The thermistor replaces the short circuit employed to achieve the impedance matching between the antenna and the chip in the initial solution. Note that this allows to maintain a similar (and so, realistic) assembling process.

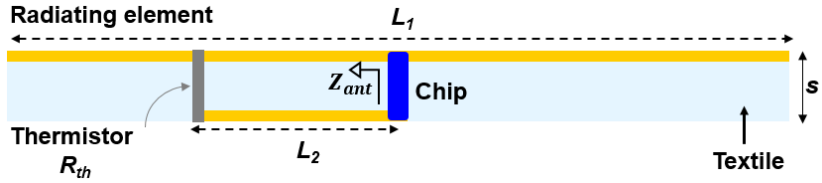

Fig. 1. Configuration of the temperature sensor-tag yarn.

\section{B. Theoretical Concept}

The temperature variation creates a modification of the thermistor's resistance $R_{t h}$ which impacts the antenna's input impedance $Z_{\text {ant }}$. This modification causes an impedance mismatching between the tag's antenna and the tag's chip that can be represented by a transmission coefficient $\tau$, expressed by (1).

$$
\tau=\frac{4 R_{\text {chip }} \cdot R_{\text {ant }}}{\left|Z_{\text {chip }}+Z_{\text {ant }}\right|^{2}}
$$

where $Z_{\text {chip }}=R_{\text {chip }}+j X_{\text {chip }}$ is the complex impedance of the RFID tag's chip and $Z_{\text {ant }}=R_{\text {ant }}+j X_{\text {ant }}$ is the complex impedance of the RFID tag's antenna.

In order to determine the transmission coefficient $\tau$ for a fixed chip impedance, the expression of $Z_{\text {ant }}$ has to be defined 
as a function of the thermistor resistance $R_{t h}$, and of the stub's length Therefore, using the transmission line theory, the loaded stub's impedance viewed at the center of the radiating element can be expressed by:

$$
Z_{t h}=Z_{0} \frac{R_{t h}+j Z_{0} \tan \theta}{Z_{0}+j R_{t h} \tan \theta}
$$

where $\theta=2 \pi L_{2} / \lambda$ is the stub's electrical length (with the $\lambda$ wavelength) and $Z_{0}$ is the transmission line's characteristic impedance considered in free space. The characteristic impedance $Z_{0}$ can be expressed depending on the transmission line's dimensions as: $Z_{0}=276 \log _{10}\left(\frac{s}{\sqrt{a a^{\prime}}}\right)$.

Finally, the antenna's input impedance $Z_{\text {ant }}$ is the result of a shunt between the radiating dipole impedance $Z_{a}$ and the loaded stub's impedance and can be expressed as:

$$
Z_{\text {ant }}=\frac{\alpha Z_{a} Z_{t h}}{\alpha Z_{a}+Z_{t h}}
$$

where $\alpha=\ln \left(s / a^{\prime}\right) /\left[\ln \left(s / a^{\prime}\right)-\ln \left(a / a^{\prime}\right)\right]$ is the step-up factor that equals 4 if $a=a^{\prime}$. It is important to note that when $R_{t h}=0 \Omega$, the sensor tag exhibits the same behavior as the matched RFID tag using the short circuit (i.e., "gammamatch" of the initial solution).

\section{SimUlation AND CHARATERIZATION OF THE RFID TEMPERATURE SENSOR-TAG YARN}

The dimensions of the temperature sensor-tag yarn have been optimized for an operating frequency in the European UHF RFID band. The final structure has the following dimensions: $L_{1}=136 \mathrm{~mm}, L_{2}=23 \mathrm{~mm}, \mathrm{a}=\mathrm{a}^{\prime}=60 \mu \mathrm{m}$, $s=0.44 \mathrm{~mm}$.

The considered Negative Temperature Coefficient (NTC) thermistor [11] is characterized by a decrease of its resistance for an increase of the temperature.

In order to study the impact of the temperature on the impedance matching of the sensor-tag in free-space, the transmission coefficient described by (1) is employed. The values of this coefficient are derived from electromagnetic simulations using the full wave simulation tool CST Microwave Studio ${ }^{\circledR}$ 2018. The thermistor's resistance $R_{t h}$ is varied between $0 \Omega$ and $22 \Omega$ to describe a temperature variation comprised between $25^{\circ} \mathrm{C}$ (ambient temperature) and a temperature higher than $150^{\circ} \mathrm{C}$. The latter condition allows to achieve the impedance matching case (i.e., short circuit at the thermistor level which brings at the chip's level an equivalent impedance which is the complex conjugate of the chip's impedance).

The obtained result shows the impedance mismatching as a function of temperature. Moreover, two resonances are observed. In the case of the matching condition (zero resistance), the first resonance is observed at $865 \mathrm{MHz}$ and the second one at $1070 \mathrm{MHz}$. These two resonance frequencies may be the result of the presence of two conductors having the lengths $L_{1}$ and $L_{2}$.

\section{EXPERIMENTAL CHARACTERIZATION OF THE RFID TEMPERATURE SENSOR-TAG YARN}

\section{A. Fabricated Prototype}

The designed RFID temperature sensor-tag yarn has been fabricated using the E-Thread $\AA$ assembling process [4]. Fig. 3 shows the sensor-tag that is integrated within a textile yarn.
The dimensions in millimeters are: $136 \times 23 \times 0.44 \times 0.06$ $\left(L_{1} \times L_{2} \times s \times a\right)$.

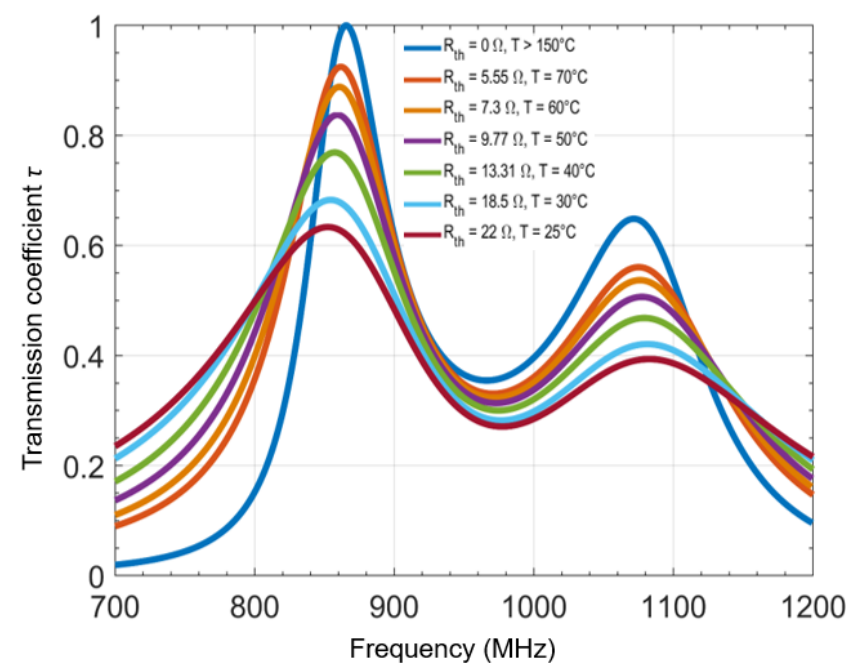

Fig. 2. Simulated transmission coefficient versus frequency for different temperature valeurs.

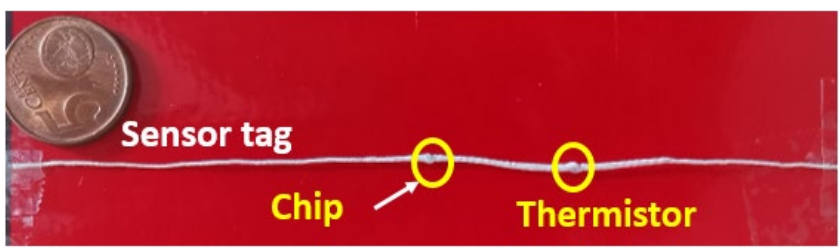

Fig. 3. Fabricated RFID temperature sensor-tag using the E-Thread $\mathbb{}$ technology.

\section{B. Experimental Setup}

Fig. 4 illustrates the experimental setup employed in order to characterize the manufactured sensor-tag prototype. The RFID sensor-tag is placed within a box and the temperature inside it is varied using a hot-gun and monitored using a thermocouple. The performance of the sensor tag is measured using Tagformance Pro test bench from Voyantic [12].

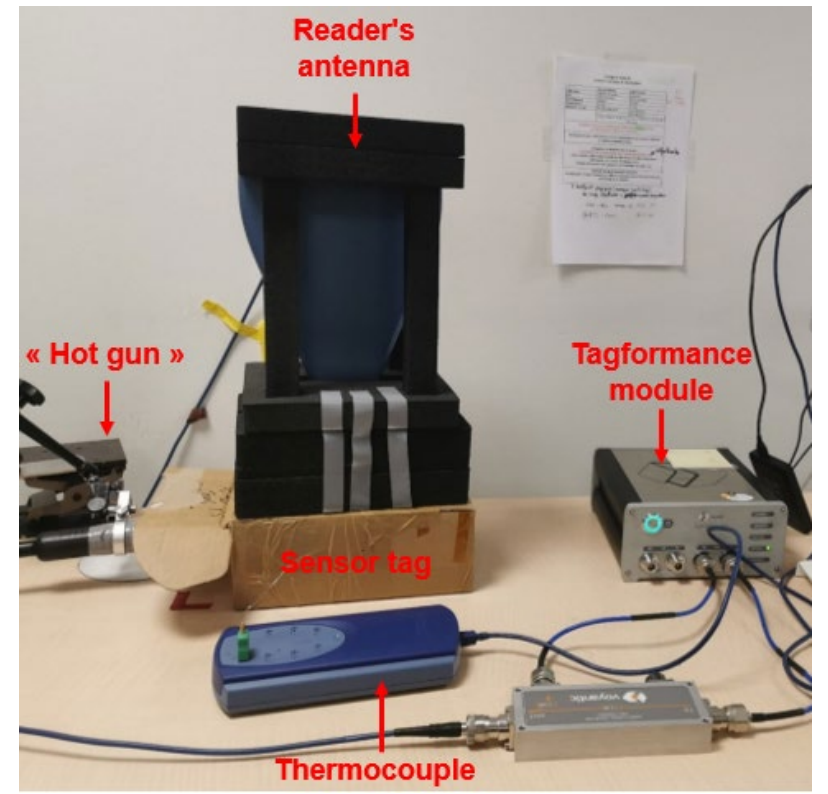

Fig. 4. Sensor-tag characterization setup using the Tagformance Pro test bench from Voyantic. 


\section{Evaluation in terms of Read Range}

The read range is an important and common criterion employed in practice for the characterization of an RFID UHF communication performance. This parameter is directly dependent on the transmission coefficient between the tag's antenna and the tag's chip. In the present evaluation, we are choosing the read range in order to highlight the tag's temperature variation. More precisely, when the temperature of the environment containing the sensor-tag varies, the thermistor's resistance is varying also and consequently the transmission coefficient follows the variation as shown by Fig. 2 . Finally, the reading range is impacted by the mismatch between the tag's antenna and the tag's chip. In practice, the activation power (i. e. the minimum power level of the signal provided by the reader which activates the tag) will vary. Indeed, when the temperature increases, the activation power will decrease because the transmission coefficient $\tau$ increases.

Using the described experimental setup, the reading range of the sensor-tag is measured for a temperature range comprised between $25^{\circ} \mathrm{C}$ and $70^{\circ} \mathrm{C}$ (not more, in order to protect the RFID chip from heating issues).

The reading range for four temperature values and in the frequency range from $700 \mathrm{MHz}$ to $1200 \mathrm{MHz}$ is shown in Fig. 5. At an ambient temperature of $25^{\circ} \mathrm{C}$ the sensor tag has the lowest reading range of 4.5 meters. The increase of the temperature increases the reading range until it reaches the value of 6.2 meters. This behavior is in coherence with the proposed concept consisting of the sensor-tag's impedance matching improvement as the temperature increases.

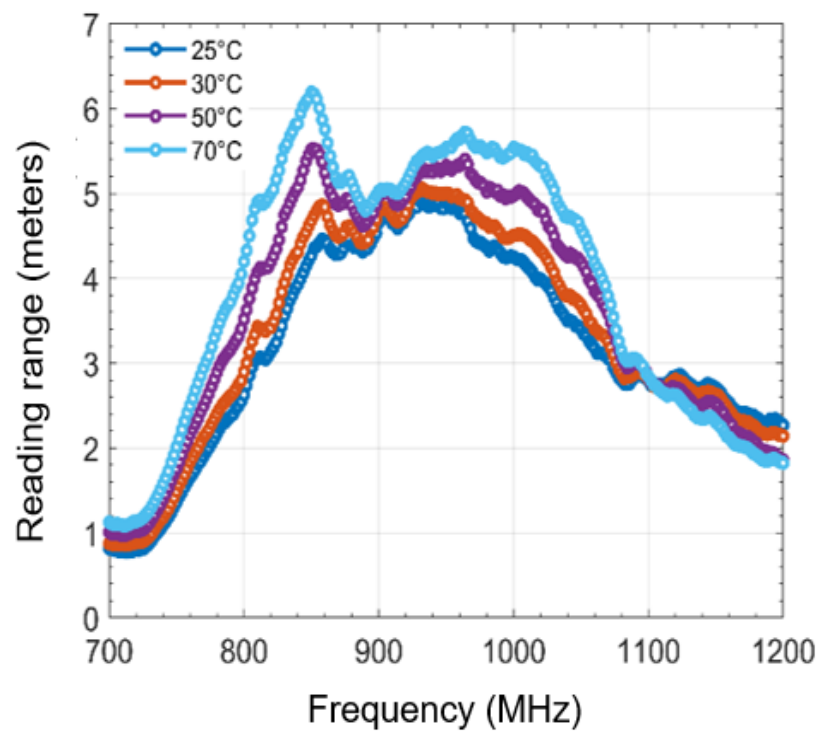

Fig. 5. Read range of the sensor-tag measured for a temperature range of $25^{\circ} \mathrm{C}-70^{\circ} \mathrm{C}$.

\section{CONCLUSION}

In this paper, a UHF RFID temperature sensor-tag has been proposed for an integration into a textile yarn using the E-Thread $\AA$ technology. By employing electromagnetic simulations, the dimensions of the tag's antenna and of the stub were optimized in order to obtain an impedance matching at $865 \mathrm{MHz}$ for a zero resistance condition. A first sensor-tag prototype has been fabricated and experimentally tested. It is demonstrated that the temperature has an impact on the reading range of the sensor-tag which validates the proposed concept. The RFID yarn integrating a temperature sensor has very promising applications for example for monitoring the temperature of a clothing wearer in a non-invasive way while maintaining the passivity of the RFID tag.

\section{ACKNOWLEDGMENT}

We would like thank the Région Auvergne-Rhône-Alpes for funding RAFTING project. Thank you to our industrial partner Primo1D for their contribution in the manufacturing process.

\section{REFERENCES}

[1] Y. Duroc, S. Tedjini, "RFID: A key technology for Humanity," Comptes Rendus Physique, Vol. 19, 2018.

[2] P. V. Nikitin, K. V. S. Rao, "Performance limitations of passive UHF RFID systems," IEEE Antennas and Propagation Society International Symposium, USA, July 9-14, 2006.

[3] S. Tedjini, G. Andia Vera, M. Zurita, R.C.S. Freire, Y. Duroc, "Augmented RFID tags," IEEE Radio and Wireless Week: IEEE Topical Conference on Wireless Sensors and Sensor Networks, US, January 24-27, 2016.

[4] R. Trangkanukulkij, T. Kim, W. S. Kim, “A 3D printed flexible passive RFID for temperature sensing," International Flexible Electronics Technology Conference (IFETC), Canada, August 7-9, 2018.

[5] J. Virtanen, L. Ukkonen, T. Bjorninen, A. Z. Elsherbeni, L. Sydänheimo, "Inkjet-printed humidity sensor for passive UHF RFID systems," IEEE Transactions on Instrumentation and Measurement, vol. 60 , no. 8, pp. 2768-2777, 2011

[6] H. He, X. Chen, L. Ukkonen, J. Virkki, "Clothing-integrated passive RFID strain sensor platform for body movement-based controlling," IEEE International Conference on RFID Technology and Applications (RFID-TA), Italy, September 25-27, 2019.

[7] G. Andia, "Slenderly and conformable passive UHF RFID yarn," IEEE International Conference on RFID (RFID), USA, May 9-11, 2017.

[8] D. Vicard, J. Brun, "Cap for a chip device having a groove, device provided with said cap, assembly consisting of the device and a wire element, and manufacturing method thereof," Aug. 13 2015, US Patent App. 14/428,254.

[9] S. Benouakta, F. Hutu, Y. Duroc, " New approaches for augmented UHF RFID textile yarn,' IEEE International Conference on RFID Technology and Applications (RFID-TA), Italy, September 25-27, 2019.

[10] Impinj. Monza 4 Product Brief. [Online]. Available: https://support.impinj.com/hc/en-us/articles/202756928-Monza-4Product-Brief

[11] Murata. NTC thermistors. [Online]. Available: https://www.murata.com/en-eu/products/thermistor/ntc.

[12] Voyantic. Tagformance Pro. [Online]. Available: https://voyantic.com/products/tagformance-pro 